\title{
The endocannabinoid system: a modulator of brain-behavioral processes
}

\author{
Sam A. Deadwyler
}

Received: 28 March 2008 / Accepted: 29 March 2008 /Published online: 7 May 2008

(C) Springer-Verlag 2008

In this special issue of Psychopharmacology, the functional significance of the endocannabinoid system is examined with respect to many different behavioral, physiological and cellular processes. Because of the ubiquity and distribution of $\mathrm{CB} 1$ and $\mathrm{CB} 2$ cannabinoid receptors in the mammalian central nervous system, delineation and assessment of the many neuronal targets affected when endocannabinoids are released is critical. In addition, it is important to know the conditions under which release occurs in order to identify circumstances where behavior, physiological processes, and/ or antecedent cognitive function may be affected. The range of investigations described in this special issue extends from rodents to humans and deals with aspects of endocannabinoid system function addressed in several different ways. Endocannabinoid systems significantly modulate appetite or use of drugs. Investigating the hypothesis that increasing endocannabinoid levels by suppressing the degradative enzyme FAAH with the compound URB597 would affect alcohol consumption in rats, Cippitelli et al. report here that the anxiolytic indicants of withdrawal from ethanol were significantly suppressed in animals receiving the compound. Also, the study by Kelsey and Calabro showed that endocannabinoids may enhance sensitization to nicotine; however, once the sensitization developed from repeated injections, endocannabinoids did not appear to be involved. These results may also relate to those reported by Jarbe et al. on discriminative stimulus properties of cannabinoid receptor antagonists, showing that a form of discriminated taste aversion was selectively sensitive to $\mathrm{CB} 1$ receptor antagonists

S. A. Deadwyler $(\bowtie)$

Physiology, Wake Forest University,

Medical Center Blvd,

Winston-Salem, NC 27157, USA

e-mail: sdeadwyl@wfubmc.edu with differing degrees of inverse agonist properties. In a different context, Gorzalka et al. showed that blockade of CB1 receptors by AM251 was effective in facilitating male sexual behavior in rats, suggesting that endocannabinoids operate similar to exogenous cannabinoids in this context. McMahon et al. show in this issue that the discriminative stimulus properties of exogenous cannabinoids, such as $\mathrm{CP}$ 55940, were distinct from methanandamide, indicating that mediation of endocannabinoid actions may be via receptors that actually differ from CB1 receptors, an issue now amenable to genetic manipulation because the studies were performed in mice. The article by Gerald et al. utilized knockout mice to examine whether presence of CB1 receptors influenced expression of dopamine and opioid receptors in striatum of young and mature male and female rats. The findings suggest that age, gender, and presence of CB1 receptors influence expression of striatal dopamine receptors. Interestingly, contrasting results are also reported in this issue with respect to the anxiolytic-like effects of cannabinoids in rodents and squirrel monkeys. Sirli Raud et al. report that expression of anxiety was associated with changes in the levels of enzymes responsible for the degradation of cannabinoids in PAG and amygdala in rats exposed to cat odor, but the study by Delatte and Paronis did not support a role for cannabinoids in suppressing benzodiazepine sensitive anxiolytic-like responding in squirrel monkeys. However, in the article by Trezza et al. rat offspring from mothers given moderate doses of delta-9-THC showed alterations in normal social behavior and vocalization and other anxiolytic-based changes that persisted into adulthood.

An important area of endocannabinoid involvement is with respect to its role in information processing in hippocampus and the influence on hippocampal-related behaviors. The retrograde influence of endocannabinoids on excitatory and inhibitory synaptic processes, now extended 
to several other brain regions, was examined with respect to the specificity of endocannabinoid modulation of GABA-A and GABA-B related systems by Lafourcade and Alger. From a behavioral perspective, Riedel et al. report that localized intrahippocampal infusion of CB1 antagonists in rats tested in the Morris Water Maze produced differential effects dependent upon state of learning and performance. The article by Rueda-Orozco et al. provides further evidence that the endocannabinoid system may mediate differentiated types of informational demands by showing an increase in expression of cDNA for $\mathrm{CB} 1$ receptors in rats using different strategies to solve a multi-choice maze task. This was also confirmed by the findings reported by Deadwyler and Hampson that endocannabinioids are only active on certain types of trials within a delay-type memory task and that systemically produced blockade of endocannabinoid function can improve performance by eliminating pre-existing behavioral biases. Finally, in a well-controlled, drug-interactive paradigm, D'Souza et al. gave extensive testing to human subjects administered haloperidol and delta-9-THC to determine the possible role of $\mathrm{D}_{2}$ receptors in cannabinoid-related psychomimetic symptoms and cognitive function. It was determined that $D_{2}$ receptors are not critical for most of cannabinoid influences in humans with respect to psychosis; however, the two drugs did interact to produced enhanced impairment on some cognitive tests, in particular, short-term working memory. The collection of papers in this special issue demonstrates that endocannabinoids are now implicated in many brain-behavior relationships due to their ability to modulate the function of a number of different neurotransmitter and cellular systems.

Acknowledgements It is with great appreciation that I acknowledge those involved in the production of this special issue of Psychopharmacology. I am especially indebted to the reviewers and their expert opinions regarding revisions and suggestions for making the articles more appropriate for this issue. I also thank all the authors for their kind acceptance of these suggestions and their efforts to make their presentations focus on the role of endocannabinoids. 